\title{
DANSE EN EPS : LES DILEMMES DU GUIDAGE VERS LA CREATION
}

\begin{abstract}
Résumé : Le but de la recherche est de comprendre comment les enseignants d'éducation physique et sportive conduisent leurs élèves à réaliser une chorégraphie en danse. Les procédures d'enseignement étudiées pour cette pratique physique artistique sont les usages et conceptions de la démonstration et des aides à création et la composition. L'étude s'appuie sur quatorze entretiens semi-directifs réalisés auprès d'enseignants dont le degré d'expertise est variable. Les résultats montrent que les démonstrations de l'enseignant et celles des élèves répondent à plusieurs fonctions mais suscitent des réticences malgré leur usage avéré. Les procédures de création sont guidées par des alternatives, hésitant entre libertés et contraintes. Ce que recouvre implicitement l'art est variable selon le niveau d'expertise.
\end{abstract}

Mots-clés : danse, EPS, création démonstration.

\section{INTRODUCTION}

L'objectif de cet article est de mettre à jour la manière dont varie, en fonction du degré d'expertise des enseignants d'EPS, l'usage de certaines procédures d'enseignement lors de séances de danse. L'article se centre sur la démonstration, puis sur les procédures conduisant les élèves à « entrer en création » (gérer composer/improviser/fixer) pour construire une chorégraphie. Enfin, il s'agit de cerner la manière dont les enseignants approchent et définissent, en fonction de leur expertise, ce qu'il y a d'artistique dans la danse (en la différenciant des pratiques sportives) ou son enseignement.

La première partie de l'introduction détaille des travaux récents qui pointent la complexité de la démonstration gestuelle et son caractère actif, contrairement à ses connotations habituelles d'imitation passive. Les décalages fréquents entre conceptions de l'enseignement d'une pratique artistique et les mises en œuvre concrètes sont ensuite rappelés.

La seconde partie resitue le contexte des activités physiques artistiques en EPS : les grandes lignes des programmes, le rappel du déroulé de la formation initiale des enseignants d'EPS, la teneur des débats professionnels consacrés à l'introduction de la danse et du cirque en EPS.

\section{Les études sur la démonstration}

Globalement la démonstration a mauvaise presse en EPS. Elle témoigne d'un passé révolu que les enseignants souhaitent mettre à distance ; elle évoque des procédures d'enseignement jugées périmées (ordre donné par le moniteur, reproduction passive des élèves) et ne laissant pas la place à ce qui semble plus conforme aux attendus d'une éducation actuelle : faire réfléchir au pourquoi et au comment, individualiser les situations d'apprentissage... La démonstration semble ainsi être particulièrement contradictoire avec le fait d'enseigner une pratique physique «artistique », en sollicitant une exécution plus qu'une «création » de la part de l'élève. Or, la recherche d'une composition personnalisée, de l'émotion 
sont présentes dans les formulations des programmes relatifs à ces activités physiques.

Pourtant les travaux scientifiques en psychologie démontrent le caractère complexe de la reproduction, de l'imitation d'une gestuelle, procédure moins simpliste qu'il n'y paraît. Lafont (2014), s'appuyant sur la théorie de Bandura (apprentissage sociocognitif par observation, ASCO), rappelle qu'on tire bénéfice de l'imitation d'autrui, particulièrement par la démonstration explicitée, c'est-à-dire associée à des commentaires. Loin d'être passif, l'imitateur doit construire un modèle interne, sélectionner et coder l'information ; des processus mnésiques, attentionnels et moteurs sont sollicités et aident à l'amélioration du sentiment d'efficacité personnelle (ou sentiment de compétence dans la tâche à réaliser). Lafont rappelle, en danse, la supériorité de la démonstration explicitée sur la démonstration silencieuse et sur d'autres procédures comme l'aménagement du milieu pour concrétiser les tâches.

Harbonnier-Topin et Barbier (2014), étudiant des cours de danse auprès d'étudiants visant à devenir professionnels, ont analysé avec beaucoup de finesse des situations de démonstration-reproduction. La démonstration se compose de quatre activités différentes :

- faire ce qu'il y a à faire : l'enseignant exécute (la démonstration au sens traditionnel).

- dire quoi faire : il commente ou nomme les éléments réalisés (« monter le bras droit, pivoter vers la droite »).

- faire comment faire : l'enseignant explicite « en action », il ralentit ou décompose le mouvement, mime les erreurs d'exécution.

- dire comment faire : il commente la manière de parvenir à reproduire sa démonstration, il fournit des informations procédurales sur le mouvement : "chercher à allonger le côté droit », "garder le poids sur le talon... ».

Du côté des étudiants qui exécutent la proposition dansée de l'enseignant, les auteurs distinguent l'appropriation de la réalisation :

- s'approprier : faire simultanément avec celui qui montre ou faire après, en décalé ; c'est aussi regarder sans faire, questionner et réfléchir sur le dire et le faire ;

- réaliser : faire pour s'exercer ou faire pour répéter et fixer.

Ainsi, les travaux sur l'intérêt didactique de la démonstration convergent pour pointer la complexité de l'appropriation d'une démonstration. Ils soulignent l'importance et la diversité des sous-activités ${ }^{1}$, en particulier celles liées au « dire » accompagnant la démonstration-reproduction. La reproduction est bien loin d'être une activité d'imitation passive.

Mais la démonstration n'est qu'une des voies possibles pour doter les élèves de séquences gestuelles, chorégraphiées ensuite dans une prestation soumise au regard des spectateurs. L'enseignant doit donc aussi guider les élèves pour aboutir à celle-ci : les choix parmi de multiples possibilités de guidage sont possibles et renvoient à des conceptions et des représentations multiples.

Enseigner une pratique artistique :

diversité des démarches enseignantes

Comment diriger une séance de danse, alors que cette pratique physique semble orientée selon un objectif d'individualisation? Lam et Kember (2006) ont

${ }^{1}$ « Activité » au sens psychologique du terme. 
cherché à approfondir la manière dont les enseignants traduisaient concrètement, dans les mises en pratique, leurs conceptions de l'enseignement artistique. Les auteurs ont identifié :

- cinq conceptions à partir de six indicateurs (objectif, nature des connaissances artistiques, du talent, de la technique et créativité, du produit et processus artistique, des résultats attendus de l'apprentissage) : trois conceptions essentialistes visant le développement moral ou artistique en art, deux conceptions contextualisées visant le développement intellectuel ou la thérapie au moyen de l'art.

- quatre approches à partir de six indicateurs (nature de ce qu'il y a à réaliser, contenus d'enseignement, rôle de l'enseignant, de l'élève, méthodes et évaluation) : les approches instructive ou de recherche disciplinaire, centrées sur la matière ; les approches visant les interactions sociales ou le développement personnel, centrées sur l'élève.

Leur enquête qualitative auprès d'enseignants de niveaux de formation divers repère six combinaisons conceptions/approches (parmi les 20 possibles) : 16 des 18 sujets mixent conceptions et approches (une conception essentialiste et une approche centrée sur l'élève, par exemple), ce qui peut apparaître a priori incompatible. Ces choix et combinaisons dépendent du niveau de formation des enseignants. L'influence des programmes officiels et de l'évaluation n'est repérable que pour les élèves plus âgés (lycée/collège). Ces travaux incitent à interroger simultanément les conceptions des enseignants, et à les analyser de manière empirique à travers des descriptifs précis des approches mises en place, pour enseigner la danse ou plus globalement les pratiques physiques artistiques en EPS. On peut alors mettre en regard ces pratiques avec le niveau d'expertise des enseignants.

\section{Le contexte des activités artistiques en EPS}

Les programmes de 1'EPS mentionnent quatre regroupements d'activités physiques en collège, cinq en lycée (activités collectives, de pleine nature, athlétiques, etc.). Chacun de ces groupes doit figurer dans le curriculum des élèves. Un de ces regroupements, assez hétérogène, rassemble sous l'intitulé général d' " activités physiques artistiques »: la danse, la gymnastique sportive, la gymnastique rythmique, les arts du cirque, l'acrosport et l'aérobic. Ces activités sont fédérées dans les programmes à travers l'objectif d'une prestation « artistique, esthétique ou acrobatique $»^{2}$. Il existe donc une possibilité pour les enseignants d'EPS de substituer n'importe quelle activité gymnique-acrobatique à une activité artistique telle que la danse. Ainsi, une enquête de 2006 portant sur les classes de la $6^{\mathrm{e}}$ à la terminale ${ }^{3}$ a établi que le volume d'enseignement des pratiques physiques artistiques en EPS est faible (Terret, Cogérino \& Rogowski, 2006).

La formation initiale en danse des enseignants d'EPS est généralement assez brève pour les non-spécialistes : elle peut être, actuellement, d'une vingtaine d'heures, souvent en $1^{\text {ère }}$ ou $2^{\mathrm{e}}$ année de licence (pratique physique, informations didactiques et techniques), complétée l'année des épreuves orales du CAPEPS par une préparation spécifique aux questions probables. Ce volume est très variable

\footnotetext{
${ }^{2}$ Exemple de formulation dans les programmes du collège en 2008 : « Réaliser une prestation à visée artistique, esthétique ou acrobatique : concevoir, produire et maîtriser une prestation à visée artistique ou acrobatique, devant un public ou un jury, selon un code ou des règles de scène en osant se montrer et s'assumer ».

${ }^{3}$ L'étude portant sur 2500 enseignants d'EPS, issus de six académies différentes, situe le volume horaire annuel consacré à la danse de $5 \%$ en $6^{\mathrm{e}}$ à $1 \%$ en classe terminale; de 1 à $2 \%$ en lycée professionnel. L'enseignement de la danse en classes mixtes varie de $95 \%$ des classes de $6^{\text {e, }}$ à $60 \%$ de celles de terminale (valeurs numériques arrondies) (Terret, Cogérino \& Rogowski, 2006, tableaux 57 et 58).
} 
selon les centres de formation et le pronostic établi par l'étudiant sur ses chances de « tomber » sur cette activité au tirage au sort des oraux. La pratique physique et les compétences d'enseignement sont donc très limitées pour certains et parfois lointaines quand l'enseignant est confronté à ses premiers élèves. Les étudiants peuvent aussi choisir une «option » pour une pratique physique de leur choix et ainsi accroître leur spécialisation relative à une pratique physique artistique. Les volontaires ont également la possibilité de s'inscrire dans les associations sportives universitaires pour pratiquer davantage. Ces deux possibilités, option et association sportive, donnent l'opportunité d'accroitre les compétences techniques, didactiques de ces étudiants, qui se spécialisent sur ces activités.

Le type de danse valorisé en formation est actuellement la danse contemporaine; le jazz et le modern jazz font partie du bagage des enseignants formés jusqu'au milieu des années quatre-vingt, ou bien de ceux qui l'ont pratiqué comme loisir personnel ; le hip hop est peu présent en formation initiale.

Du milieu des années soixante au milieu des années quatre-vingt, l'« Expression corporelle " s'est largement substituée à l'enseignement de la danse en EPS, avec le rejet de la «technique » et la mise à distance de la recherche d'efficacité motrice (Arguel, 1992 : 43-44). Ces deux rejets ont suscité de vifs débats : désarçonnés face à une activité jugée « sans contenus » et sollicitant des " débordements » émotionnels, de très nombreux enseignants ressentaient l'impossibilité de guider les apprentissages des élèves (absence de démonstration et imitation gestuelle ; situations exploratoires, de recherche) et craignaient ainsi la perte de leur statut d'intervenant. Le remplacement du terme «ExCo » par « Activités Physiques d'Expression » puis «Activités Physiques Artistiques » (dans les années quatre-vingt-dix) a offert une nouvelle opportunité aux enseignants : viser de nouveau des apprentissages spécifiques, relatifs à la gestuelle et aux processus de création. Cependant la grande latitude concernant les procédures d'enseignement pour ces activités perturbe toujours nombre d'enseignants d'EPS, en difficulté pour mettre en place et guider des situations de recherche (motrice ou chorégraphique) (Lemonnier, 2014).

Actuellement, la nature artistique et non-sportive de la danse est un frein notable à son enseignement en EPS. Les enseignants d'EPS sont toujours fortement impliqués dans une pratique physique personnelle compétitive et jouent également des rôles actifs dans l'organisation du mouvement sportif en club (Benhaïm-Grosse, 2007). Cet habitus sportif les éloigne des activités physiques artistiques : la morale de l'effort, le goût du dépassement de soi, l'attrait de la performance, typiques de l'habitus sportif des enseignants d'EPS, s'associent plus logiquement au réinvestissement possible des prouesses gymniques et acrobatiques lors de l'enseignement du cirque. Garcia (2013) démontre les conditions de ce réinvestissement et ce transfert des ressources sportives antérieures des enseignants d'EPS. Le cirque donne l'illusion de surmonter les obstacles à l'entrée en danse chez les élèves, particulièrement les garçons, réticents face à la dimension expressive (Garcia \& Vigneron, 2006). Simultanément artistique (construction d'un numéro, de la scénographie) et acrobatique (prouesse, virtuosité, performance), en fonction des objets, supports ou « spécialités » (jonglerie, trapèze, clown, ...), le cirque permet de limiter l'engagement vers la danse, tout en souscrivant à l'obligation d'enseigner en EPS une pratique physique artistique, comme le prescrivent les programmes (Garcia, 2007). Il permet également de tenir à distance l'expressivité corporelle, pourtant constituante d'une pratique physique artistique. Cette expressivité qui fonde les représentations de la danse scolaire n'est plus celle, redou- 
tée, de l'expression des émotions et s'investit davantage dans un jeu portant sur le personnage (clown, par exemple).

Ainsi, l'association d'une formation assez brève et d'un habitus sportif bien ancré conduit des enseignants d'EPS à se montrer réticents face à l'enseignement d'une activité telle que la danse, qui leur paraît " trop artistique » (sous-entendu : « pas assez sportive »). De fréquentes incertitudes concernent les modalités d'enseignement de la danse : comment enseigner une activité longtemps dite «sans contenus », quelles procédures d'enseignement, quels modes de guidage pour aller vers une «création »?

Il s'agit dans cet article de comprendre comment les enseignants d'EPS, en fonction de leur degré d'expertise en danse, guident progressivement leurs élèves pour réaliser une chorégraphie dansée. Deux types de procédures d'enseignement sont étudiés : les conceptions et usages de la démonstration d'une part, les aides à la création et à la composition en danse d'autre part. L'ensemble de ces données concerne l'enseignement obligatoire : l'enseignant d'EPS y est le seul intervenant, sans accompagnement d'artistes, les élèves ne sont pas nécessairement « volontaires » pour ces activités qui se déroulent pendant l'emploi du temps hebdomadaire habituel. Enfin, la manière dont les enseignants approchent et définissent implicitement l'art et ce qu'il y a d'artistique dans la danse ou le cirque, sera détaillée.

\section{METHODES}

Le corpus analysé s'appuie sur des entretiens semi-directifs conduits auprès de 20 enseignants d'EPS, concernant globalement l'enseignement du Sensible en $E P S$, dont 14 spécifiquement consacrés à la danse et au cirque (annexe 1). Les sujets se différencient en fonction de cinq principales variables : leur âge (de 24 à 60 ans) et leur ancienneté professionnelle, l'établissement d'exercice (huit collèges, trois lycées et trois lycées professionnels), le volume horaire enseigné en danse ou cirque aux élèves et le sentiment de compétence dans ces activités. Au sein de chaque type d'établissement (collège, lycée), il n'a pas été fait de distinction selon le type de classe concernée. Le nombre de classes de même niveau ne permet pas des comparaisons fiables. Toutes les personnes interrogées ont suivi une formation durant leur cursus pour ces activités, complétée pour certains par des formations volontaires ou des itinéraires de pratique physique personnelle : le niveau d'expertise (pratique physique et enseignement) est donc variable selon les sujets. L'intensité de la spécialisation/expertise de chaque enseignant s'appuie sur deux éléments : le type de formation initiale (choix ou non d'une " option » de pratique pour une des activités physiques artistiques), la « quantité » d'enseignement de ces activités avec les élèves (nombre de séances conduites sur une année). Les trois niveaux d'expertise dont on a apprécié l'intensité apparaissent donc dans l'annexe 1 (non/moyenne/oui).

Les pseudonymes choisis permettent de repérer le degré d'expertise des enseignants : prénom commençant par E (les plus expérimentés), par M (expérience moyenne), par P (peu d'expérience), par C (n'enseigne que le Cirque), par R (ni Cirque ni Danse).

Les questions visaient à identifier les objectifs précis et les procédures de guidage des élèves pour les enseignants d'EPS qui enseignent la danse ou l'énon- 
cé de ce qu'il faudrait faire ou ne pas faire pour ceux qui ne l'enseignent pas. D'autres thématiques abordées dans l'entretien ne peuvent être développées ici ${ }^{4}$.

Les données ont fait l'objet d'une analyse de contenu thématique, de type inductif. Tous les entretiens transcrits intégralement (corpus de 242700 caractères) ont été découpés en items sur la base thématique, puis regroupés a posteriori et progressivement en catégories plus larges (ex : Formation : descriptifs, souvenirs positifs, négatifs, etc. ; Démonstration : exemples, justifications, difficultés rencontrées, etc.). Par ailleurs, le comptage de 75 termes typiques a été effectué à l'aide de la fonction Rechercher au sein de chaque entretien. Le sigle «* » permet de repérer les racines communes à plusieurs termes (par exemple : $\operatorname{compos}^{*}$ er, *ition signale que les deux termes composer et composition ont fait chacun l'objet d'un repérage quantitatif).

\section{LES DILEMMES VECUS DE LA DEMONSTRATION}

\section{La démonstration de l'enseignant}

\section{Dénégations initiales}

Lorsqu'on pose explicitement la question de la démonstration des enseignants, ce sont d'abord des dénégations qui s'énoncent: "moi je ne fais pas de démonstration » (Éric) ; " moi personnellement je l'utilise pas du tout 》 (Marie) ; " moi ça m'est déjà arrivé de faire un cycle complet sans jamais, jamais, jamais me mettre en scène " (Martin).

La démonstration de la part de l'enseignant est ensuite reconnue mais nuancée, minimisée : "j’le fais quelques fois sans musique, des p'tites variations au sol ou debout, hein, mais très vite on commence ensemble " (Marie) ; "quand on fait les variations je l'utilise un peu " (Marie- ève ève); " j'vais effectivement, pourquoi pas, proposer une petite phrase très typée " hip-hop 》" (Martin) ; "Je le fais ouais. Si si, sur des petits mouvements » (Éric) ; " alors je pense notamment au travail du duo, le travail du passage au sol : souvent j'le fais une fois, et puis j'dis après " à vous maintenant " " (Marie-Eve).

Une seule enseignante, expérimentée, précise qu'elle utilise d'emblée la démonstration de manière délibérée : "ça va faire dresser les cheveux sur leur tête à plusieurs collègues- mais moi je pars sur du chorégraphié, c'est à dire un enchaînement. On apprend un enchaînement mais l'élève est concentré sur la mémorisation du mouvement, mais est beaucoup moins concentré sur "qu'est-ce que je vais faire? que vont dire les autres ?". Non, on est tous en ligne. C'est un cours comme moi j'ai vécu pendant des années en modern-jazz. On part sur la musique, on la remet à zéro. On apprend le mouvement, des phrases de huit temps et voilà... » (Édith).

Passer le cap des dénégations, la suite des entretiens permet d'identifier plusieurs fonctions accordées par les enseignants à leur démonstration.

Les fonctions avérées de la démonstration

Pour mieux saisir le rapport des interviewés à la démonstration, il faut repérer les fonctions qu'ils lui accordent et les liens tissés avec la thématique de la

\footnotetext{
${ }^{4}$ Des descriptifs des procédures d'apprentissage et de formation vécues lors des FI et FPC, des ressentis à leur égard ; la formulation de leurs représentations des activités artistiques enseignées en EPS, particulièrement danse et cirque : « image » de ces activités, intérêt(s) à les enseigner en EPS, éléments qui pourraient conduire à les intégrer ou exclure de l'EPS proposée à leurs élèves.
} 
compétence. La démonstration est associée à plusieurs fonctions, où didactique et pédagogie sont étroitement tissées :

- dédramatiser, comme dans l'extrait précédent, reproduire une gestuelle imposée évite de se questionner sur ce que les autres élèves vont en penser. Il s'agit de «lancer » le travail, l'envie de faire des élèves, de lever leurs réticences initiales à l'égard de la danse ou de leur incompétence supposée : " je pense que la mienne [ma démonstration] est essentielle dans la prise de confiance, dans la mise en confiance des élèves » (Édith);

- aider les élèves à " oser », vaincre l'impression de ridicule : " quand c'est des ateliers, que je les sens plus réticentes ou que c'est plus facile.... Et puis je pense que m'engager moi corporellement devant elles, c'est aussi les aider à oser le faire après, quoi " (Marie-Eve) ; "la réticence..., c'est une activité où il faut oser. Pour que les élèves démarrent, il faut que le prof montre contrairement à d'autres activités » (Rita) ; "je veux leur expliquer, à travers ce que moi je démontre, qu'on ne danse pas en se représentant soi, mais on danse pour représenter un personnage » (Édith).

- rendre concret, compréhensible ce qui est attendu d'eux, clarifier les exigences : "l'idée étant vraiment de leur montrer que plus j'vais faire le geste "à fond" en mettant de l'intensité, de la vitesse, en jouant sur toutes ces variables, plus j'vais réussir à être dans le propos et l'évoquer" (Pierre) ; "leur faire comprendre qu'ils ne font pas exactement le même et qu'il faut bien placer son bras là, parce que le bras doit être là pour l'ensemble du groupe, ben oui je le fais » (Éric) ; "c'est plus comme un inducteur, c'est donner une idée de comment on peut faire [...] J'ai l'impression que ça aide à la compréhension de c'qu'il y a à faire » (Marie-Eve).

Le décalage de compétences entre l'enseignant et sa classe est aussi perçu comme un risque de désengagement des élèves fragiles, peu confiants. Pour cette raison, un enseignant expérimenté préfère "passer des modèles de Danse de Youtube ", renonçant à faire lui-même des démonstrations : "qui pourraient écraser et où ils pourraient se sentir en comparaison, ce qui ne serait pas bénéfique pour eux» (Éric). On constate aussi que les deux plus expérimentés (Éric et Édith) adoptent, pour mettre les élèves en confiance, une attitude diamétralement opposée quant à l'usage de la démonstration de l'enseignant, reliant leur position aux spécificités de leur public d'élèves.

- assurer sa crédibilité : " mouiller sa chemise », en démontrant, est explicitement formulé par plusieurs enseignants. Il faut gagner la confiance des élèves, les "épater » un peu, pour asseoir sa crédibilité : « si l'enseignant se mouille pas, je pense que les élèves adhèrent moins. [...] Le fait que l'enseignant démontre, le fait qu'il soit avec eux, ça facilite le regard des autres, je pense " (Pierre); "qu'ils s'aperçoivent que moi, je me mouille avec eux et que, des fois, je fais des trucs complètement farfelus, mais ça fait partie de la mise en scène " (Édith). Ici le propos évoque le fait que "se lâcher », oser faire du «farfelu» est bienvenu, voire souhaitable, dans une activité artistique.

Démontrer face aux élèves, c'est aussi dévoiler à leurs yeux le niveau de compétence motrice de l'enseignant en danse, niveau variable selon le type de formation plus ou moins approfondie.

Démontrer sans se montrer ?...

Ainsi montrer aux élèves qu'on est plus compétent que la classe met en difficulté les enseignants non spécialistes : «moi, pour être franche, il y a des activités où je ne démontre rien à mes élèves. Je les mets en action sans avoir besoin de 
montrer. [...] Comme en danse, je pense qu'il faut montrer et mouiller la chemise, pour que les élèves se lancent, je pense que oui, il faut avoir vécu, plus que dans les autres activités, l'activité pour l'enseigner» (Rita).

Cette démonstration suscite des réticences issues de l'histoire des styles d'enseignement de l'EPS au $\mathrm{XX}^{\mathrm{e}}$ siècle. L'uniformisation des élèves, la planification formelle des apprentissages sont l'objet de forts rejets, d'autant plus accentués que la danse draine en EPS l'image d'une activité où devrait s'imposer l'individualisation des personnes, l'expression de leur singularité. Ce positionnement défensif est clairement énoncé chez les enseignants non-spécialistes des activités artistiques : " si on montre un geste, après on peut être sûr que toute la classe fera le même geste dans sa chorégraphie [...] Le problème de leur montrer le mouvement, ce qui me gêne, c'est par rapport à la notion de créativité, quoi » (Rémi). Alors que les spécialistes n'hésitent pas à démontrer pour « faire comprendre » rapidement, (ou « faire comment faire ») ce qu'il y a à faire, les non-spécialistes récusent cette possibilité : "c'est vrai que si je leur montre, tout de suite ils vont comprendre ce qu'il faut faire » (Rémi).

\section{La démonstration des élèves}

Demander aux élèves une démonstration est une pratique courante. Les fonctions qui lui sont accordées dans la pratique touchent à la fois à l'engagement des élèves dans la tâche, la progression dans ses apprentissages de danseur, de chorégraphe et de spectateur. Son usage est associé à la crainte d'une certaine stigmatisation possible, en fonction du degré d'expertise de l'enseignant.

Pour le danseur ou le spectateur, pour donner envie ou faire progresser ?

La démonstration effectuée par les élèves n'est jamais abordée d'emblée, mais elle est utilisée au service de plusieurs fonctions relatives à la confiance en soi, la confiance réciproque et à la progression du travail.

- mettre les élèves en confiance : montrer aux autres élèves ce à quoi on a abouti au terme des séquences de travail accroît la confiance en soi : passer devant les autres emplit de fierté le groupe par exemple. Cette épreuve rassure sur ses propres compétences ;

- les rendre " actifs » et non plus « imitateurs" : "c'est vrai que des fois on se repose sur le modèle, c'est moins fatiguant de se laisser conduire... On en a besoin, donc faut jouer là-dessus quoi, on fait une proposition, les élèves se l'approprient et après c'est eux qui sont moteurs, c [e n]'est plus le prof qui est moteur » (Marie) ;

- baliser les étapes franchies ou à venir, suggérer aux élèves que leurs trouvailles méritent d'être échangées avec toute la classe, en leur montrant que c'est aussi eux qui font avancer le travail en assumant la topogenèse des savoirs. Pierre souhaite que les élèves puissent constater la diversité de leurs procédures gestuelles à partir d'un même inducteur (ex : illustrer corporellement un verbe d'action). Pour Marie-Eve, " quand j'vois des façons qui me plaisent, qui m'attirent, qui m'intéressent, et bien j'dis "allez, chacun va me montrer quelque chose " ou dire "regardez une telle, elle l'a fait " ou parce j'ai donné l'atelier et que l'élève crée quelque chose que j'voulais faire plus tard».

Les interviewés semblent ne pas suivre de projet délibéré à propos de la démonstration des élèves : ils saisissent de manière impromptue les opportunités, en fonction du contexte relationnel, motivationnel et du projet qu'ils ont pour la suite des séances. Ils réagissent bien plus, en rebond, qu'ils n'appliquent une stratégie explicite. 
- constater l'utilité des exigences formulées : Éric met en place des démonstrations pour que les groupes comprennent qu'il y a parfois des exigences à respecter (par ex : être «synchro », effectuer tous exactement la même gestuelle) : ici les groupes spectateurs peuvent renvoyer des commentaires sur le respect des exigences formulées dans les consignes initiales de la situation, sans que ce soit toujours l'enseignant qui pointe ce qui pourrait être affiné dans l'exécution gestuelle.

- forger le jugement: il s'agit de passer devant les autres pour montrer l'état final (parfois temporaire) des situations de recherche. L'accent est alors mis sur l'activité des observateurs, les «spectateurs », sur la nature des repères à utiliser pour décrire, commenter ou analyser une chorégraphie. La stratégie de l'enseignant est délibérée, la démonstration devient un rituel, une balise : "C'est davantage pour être observateur de ce qu'ont fait les autres, être indulgent, être dans la lecture: "qu'est-ce qu'a voulu exprimer votre camarade en faisant tel mouvement? ", sur les ressentis, les émotions, mais pas du tout sur la technique [...] On ne peut pas être danseur et chorégraphe si on n'a pas appris à être spectateur. Donc il faut qu'il y ait des démonstrations. Spectateur pour avoir un regard averti, pour être respectueux de ce que fait l'autre (avec des fiches ou non), et voilà ça c'est important. Donc dès le début du cycle il y a de la démonstration " (Édith). Dans ce cas, la démonstration devant le reste de la classe n'est jamais suivie d'une imitation ou reproduction de la part des autres élèves. Elle fonctionne comme une propédeutique à l'acceptation du regard d'autrui et une étape de construction $\mathrm{du}$ regard averti.

Les risques : moquerie et mise en concurrence

Mais les dangers de la stigmatisation ou de l'assignation de rôle sont présents et formulés par les enseignants moyennement expérimentés : "Prendre un élève et lui demander entre guillemets de faire le travail du prof, de faire la démonstration devant tout le reste de la classe, ça peut l'mettre en situation pas forcément très confortable, euh il peut subir des moqueries, il peut s'rater " (Martin). Éric, plus expérimenté, adopte le parti de limiter la démonstration d'un seul élève. Il préfère s'inclure dans le groupe qui montre aux autres, afin de limiter les comparaisons auxquelles se livreraient ses élèves pour éviter que la classe s'imagine "que tel élève serait meilleur qu'un autre, parce qu'encore une fois c'est un public particulier et je n'ai pas envie de les mettre en concurrence entre eux... donc pour éviter la comparaison » (Éric).

Ainsi, la démonstration des élèves, tout comme celle des enseignants, navigue entre plusieurs dilemmes : mettre les élèves en confiance et en activité ou servir de prétexte à la formation du spectateur, donner envie de faire ou permettre de faire le point sur les apprentissages (en cours ou à venir).

La démonstration en danse : un noud de contradictions...

En synthèse, revenons sur les apports potentiels de la démonstration aux apprentissages, rappelés par Lafont (2014) : procédé actif d'apprentissage, sollicitant une sélection d'informations, aboutissant à la construction d'un modèle interne du mouvement, s'appuyant sur des processus mnésiques, attentionnels, motivationnels et de reproduction motrice. Les descriptions des interviewés évoquent souvent des esquisses gestuelles, fugitives (sauf Édith), rarement des « démonstrations explicitées ». Peu de commentaires détaillés - «dire quoi faire » et «dire comment faire »- accompagnent les démonstrations ; ils apparaissent plutôt improvisés et fugaces. Les bénéfices pointés sont plus souvent motivationnels 
(« donner confiance »); les bénéfices attentionnels ou mnésiques ne sont évoqués que par les enseignants les plus expérimentés.

Les réticences mentionnées par les non-experts renvoient à la thématique de «l'initiative » (qui est source du mouvement, de la gestuelle ?), indépendamment des bénéfices potentiels sur les apprentissages. Bien souvent, l'opportunité d'acquisition de repères techniques, moteurs ou kinesthésiques passe au second plan (repérage et stabilisation des gestuelles en vue de créer un effet, modifications posturales, coordination ou synchronisation motrice, dissociation segmentaire...). Ainsi, les thématiques du pouvoir (qui impose quoi ?) et de l'image de soi (quel regard d'autrui sur ma prestation ? ne pas se sentir ridicule...) trament les préoccupations des enseignants et leurs réticences face à la démonstration de l'enseignant.

Le terme démonstration, parfois associé à la seule «mostration » (nonsuivie d'une tentative de reproduction), fait obstacle à la création pour les moins expérimentés dans le domaine des pratiques artistiques en EPS, à l'opposé de ce qui y serait attendu : l'expression pure de l'individualité. Cette opposition est nettement abandonnée chez les plus expérimentés. Le dilemme est donc le suivant : la démonstration est antinomique de la danse, de la créativité car elle suggère la copie, la reproduction, mais elle est indispensable pour asseoir la crédibilité de l'enseignant auprès des élèves et vaincre leurs hésitations à se lancer; danser semble le pur produit de la créativité individuelle mais démontrer et demander ensuite de reproduire, ou " copier», reviendrait à nier l'individualisation de la production chorégraphique, dont l'enseignant se protège par ailleurs. L'identification de ces tensions permet de comprendre (partiellement) pourquoi les enseignants les moins à l'aise avec la danse choisissent de proposer le cirque à leurs élèves, de manière à pouvoir justifier qu'ils leur ont effectivement proposé des pratiques physiques artistiques, tout en ayant la possibilité de valoriser leurs propres ressources motrices, sportives, acrobatiques inscrites dans leur habitus et hexis corporel.

\section{L'ENTREE DANS LA CREATION :}

\section{TENSIONS ENTRE LIBERTES ET CONTRAINTES}

Une pratique artistique semble difficile à dissocier de l'idée de création, au sens d'objet final : une chorégraphie, une prestation, un évènement (happening, accumulation, exposition). Mais la création en tant que processus est susceptible d'emprunter des voies très diverses (Lam \& Kember, 2006), et tous les interviewés évoquent le fait qu' "on ne crée pas à partir de rien ». En revanche, ils divergent sur les voies empruntées pour amener leurs élèves à une création. Cette dernière est envisagée par tous comme une prestation anticipée, programmée, construite (la chorégraphie, le numéro de cirque). Aucun ne s'appuie sur des courants artistiques valorisant l'impromptu, le réactif. Par exemple le terme "improvisation » n'est utilisé que 1 fois (Édith) dans l'ensemble de tout le corpus (enchaîn*er *ement : 7 fois ; compos*er *ition : 3 fois (par 3 sujets différents) ; variation : 24 fois (dont 21 fois pour Marie-Eve) ; atelier : 34 fois (dont 14 par Marie-Eve); chorégraphie : 64 fois). Cette forte homogénéité sur la "choré » est probablement due aux choix effectués dans les programmes officiels de l'EPS pour les modalités d'évaluation au terme du module d'enseignement (une chorégraphie collective en danse, un numéro collectif en cirque).

Deux tendances fortes et opposées sont repérables : les tenants d'un guidage lâche et ceux privilégiant l'utilisation de contraintes inductrices. 


\section{Laisser libre pour accéder à l'" authenticité "}

Le guidage lâche est repérable chez les enseignants peu ou moyennement expérimentés, comme l'illustre Pierre : "l'idée c'est qu'ils soient, qu'ils expriment, qu'ils aillent vraiment sur le pôle créatif; donc moins on va les guider, plus ça va refléter leur travail. Après, des groupes qui n'y arrivent pas, qui bloquent, là j'vais les guider un p'tit peu plus ». Martin précise que les « matériaux » sont l'affaire de l'élève, sinon il n'y a pas «création ": "Au niveau de la matière c'est les élèves qui la créent pratiquement à $100 \%$, c'est à dire les mouvements, les attitudes, les postures... tout ce qui est matière, tout ce qui est utilisation de son corps au niveau espace intime, espace proche, c'est l'affaire de l'élève ".

Le point de départ peut être aussi : "une petite variation que je leur impose, avec un passage au sol, des changements d'énergie, des fermetures-ouvertures, et après ils enrichissent" (Marie). Cet extrait illustre une conception de la création à laquelle on devrait aboutir : celle qui juxtapose les différentes figures, ou actions motrices, considérées comme incontournables d'une prestation dansée « complète » (tours, sauts, passages au sol...).

Dans cette forme de gymnisation de la danse il n'y a pas à rechercher un propos, une thématique, un effet à susciter pour le spectateur.

\section{Guider pour permettre des progrès}

L'utilisation de contraintes pour générer des matériaux est le choix des plus expérimentés: "mais création dans le cadre de recherche d'ateliers, guidés. C'est guidé, c'est pas "faites quelque chose". On peut pas créer de rien " (MarieEve). Édith détaille le même point de vue en soulignant les évolutions temporelles dans son approche : "Je pense que si je les laissais libres dès le départ, là ils seraient en difficulté [...] Apprendre une chorégraphie par cœur pour les élèves en début de cycle, c'est un soulagement et pas une contrainte. A l'inverse, si je leur donnais le thème du vent et que je leur disais "débrouillez-vous avec"; là ils seraient en pleine difficulté je pense ". Dans la même veine, Éric développe l'idée qu'il y a une dialectique contraintes-libertés pour faire cheminer les élèves vers la création et que la nature des unes et des autres varie selon le public d'élèves (confiants, craintifs, en manque d'estime de soi ou prêts à toutes les surprises...). Il revient sur ses élèves de LP, en difficulté sociale et culturelle : "Pour ces élèves-là, il faut que ce soit contraignant des fois. Ça passe par des zones de passages obligatoires. Parce que justement, pour en revenir à la représentation [de la danse], ces élèves-là n'en n'ont pas du tout pour $80 \%$. Ils ne savent pas ce que c'est pour le public que j'ai, et le peu qui savent, c'est un truc très négatif: c'est pour les fous. Leur demander de créer, ce serait les mettre dans la fosse aux lions, et ce n'est pas du tout accessible pour eux; donc c'est vraiment des contraintes liées à l'espace ». Le propos d'Eric valorise le fait d'imposer un cadre (ici sur la manière de gérer l'espace) pour que les élèves puissent rechercher des trajets, des interactions, des gestuelles, des rythmes, qu'ils pensent en accord avec le thème donné à la chorégraphie ou l'atelier.

Cette dialectique contraintes/explorations possibles est l'élément majeur de différenciation entre enseignants expérimentés ou non. La crainte presque maladive des moins expérimentés est donc logiquement le dirigisme : "Après, là j'me bute à cette difficulté : c'est de pas être dirigiste, de pas leur dire c'qu'il y à a faire ». Connell (2009) relève des conceptions similaires au Royaume-Uni ${ }^{5}$.

\footnotetext{
${ }^{5} 65 \%$ des enseignants d'EPS associent danse et créativité, 94\% conçoivent la danse comme une opportunité d'être créatif au plan physique, quel que soit leur sexe et nombre d'années d'expérience. Le mot créativité est le plus fréquent du corpus, plaisir et expression sont les deux suivants (bien avant
} 


\section{Composer : explorer ou fixer?}

Dans les procédures d'enseignement évoquées par nos interviewés, l'usage des termes créer, création ne permet pas de distinguer « explorer, repérer, diverger » de « formaliser, agencer, construire, affiner », qui relèvent de deux phases distinctes du processus de création.

« Composer », à la suite des situations initiales, est apprécié différemment en fonction du niveau d'expertise de l'enseignant et s'associe à des procédures différentes. Les enseignants moins expérimentés mettent l'accent sur la diversité des possibles. Marie propose au bout de deux à trois séances une « variation » (un petit enchaînement dansé) puis souligne la multitude de possibilités de modifications : "ils s'aperçoivent que cette petite variation, on peut la mettre sur des rythmes différents, on peut y mettre de l'énergie différente, on peut la concevoir différemment, c'est à dire faire tous ensemble, on peut faire en décalé, on peut faire en cascade, on peut faire en style couplet-refrain, etc. ». La multiplicité des paramètres sur lesquels on peut jouer prend une forme insistante, sans que ces possibles soient référés à un but, occultant la possibilité de jouer de manière délibérée sur certains aspects (ou paramètres) pour susciter un impact particulier sur les spectateurs (un thème, des personnages, une atmosphère, une impression globale).

Martin «apporte des techniques» parce que «ça peut pas tomber $d u$ ciel », tandis que Marie-Eve « incite » à modifier un paramètre ou un autre. Marie déplore que dans la création finale ses élèves (sans doute submergés par toutes les possibilités évoquées) n'exploitent pas ce qui avait été élaboré précédemment : « La difficulté euh... c'est de passer... ils créent des choses à chaque fois, moi j'ai une forme d'émotion, des choses très très belles, euh et puis après, quand on va passer à une création pour euh la fin du cycle, c'est comme si des fois ils avaient oublié ce qu'on avait fait, ce qu'ils avaient fait. Ils repartent pas forcément.... Alors tout notre travail... ».

Seuls les enseignants plus aguerris détaillent la gestion temporelle de leurs interventions au cours des temps de composition de la part des élèves : " J'attends vraiment que eux se soient lancés dans l'activité, pour qu'après moi j'apporte quelque chose. Quelques fois, je leur apporte du contenu dansé mais pas du tout en début de cycle. J'attends vraiment qu'il y ait les premières appréhensions, les premières timidités qui tombent pour vraiment amener les choses dans lesquelles ils ne s'enfermeraient pas en début de cycle » (Éric).

Pour éviter que les élèves soient bloqués par le regard d'autrui ou un sentiment d'incompétence, le bagage technique apporté par l'enseignant joue le rôle de «bouée », et simultanément culpabilise les non-spécialistes de danse. Leur guidage vers la création est donc plus aléatoire, au rebond, donnant l'impression d'une absence de pilotage, d'un défaut de stratégie délibérée autre que celle de «l'exploration des possibles». Ils sont écartelés entre deux définitions de la danse : la danse c'est "permettre de se rendre compte de ce que son corps est capable de faire », et son «but est de transmettre un message » (Carine). Ils oscillent donc entre fournir un soutien (car «on ne peut pas créer à partir de rien») mais rester dans certaines limites car, si le soutien est trop appuyé et visible, dans ce cas les élèves ne créent plus mais « reproduisent» ou « appliquent ».

performance, relation, travail technique). 


\section{CARACTERISER L'ARTISTIQUE}

Cerner ce qu'on entend par «Artistique » conduit à des opinions contrastées en fonction du niveau d'expertise : l'art comme expression d'une production intime ou occasion d'un travail collectif, la technique comme masque ou outil incontournable?

Pour les enseignants qui n'enseignent ni danse ni cirque, l'art se définit par la notion de spectacle "où on peut vraiment avoir des émotions" (Rita), par la «mise en chorégraphie » (René). Il s'agit de produire quelque chose, «créer personnellement, donc [...] c'est SON identité, SA personnalité », ce qui conduit à « se mettre à nu devant tout le monde » (René). L'intérêt d'enseigner des pratiques artistiques réside dans la sollicitation des relations, de la communication entre élèves : produire en groupe permet de lutter contre l'individualisme qui imprégnerait notre société (René). Toutefois, la subjectivité des acteurs-danseurs, " la part émotion ", doit être limitée car difficile à noter, peu quantifiable (Rita). Pour Rémi, moins prolixe, la danse permet de "développer l'émotivité de l'élève, [...] sa sensation artistique », d'élargir sa culture, sans que l'on parvienne à cerner ce que recouvre le terme « artistique ».

Pour ceux qui enseignent uniquement le cirque, l'art est l'opposé de la prouesse technique et sportive. Entrer par la dimension artistique en cirque conduit à introduire un scénario, un jeu d'acteur où la technique est mise au service de choix. Pour Carine la danse est certes artistique mais aussi une activité sportive comme une autre, car elle demande « de la rigueur, de l'entraînement, de la répétition, une justesse technique ». Pour Charles, "la volonté de transmettre un message, [...] de faire ressortir quelque chose au spectateur » est l'apanage de l'art. Il déplore donc que des enseignants moins assurés en cirque se replient sur des contenus techniques et acrobatiques, et moins «sur le volet esthétique », épaulés par une fiche qui rassure, listant les niveaux de pratique, les manipulations d'objets à effectuer.

Pour les plus expérimentés, la technique en cirque est "super-gênante " parce qu'elle permet de se cacher en "oubliant complètement l'expression », alors qu'en danse, une thématique associée à une gestuelle adaptée conduit à "une vraie expression qu'il n'y a pas en cirque » (Marc). Emma trouve le cirque actuel plus artistique " avec des effets de costumes, d'histoire, [...] c'est plus dansé ». Elle souligne la nécessité de parler précisément d'un type de danse pour disserter de l'artistique : pour faciliter le contact de ses élèves (de LP) avec la danse, elle commence par du rock acrobatique, ou par du mime, dont elle induit progressivement des transformations gestuelles «pour que cela devienne de la danse ». Pour elle, c'est l'abstraction qui différencie la danse du cirque et confère un aspect artistique. Elle est la seule à poser explicitement la question de la pratique de référence au sein «DES» danses pour discuter de ce qui y serait artistique ou non : les autres interviewés font implicitement référence à la seule danse contemporaine et ceci indépendamment des sous-courants qui y cohabitent.

\section{CONCLUSION}

Dans notre enquête, le sens attribué par les enseignants à l'art est très loin du travail réel des artistes, tantôt besogneux, répétitif, tantôt ingrat et douloureux. Sizorn (2008) pour le cirque, Sorignet (2008) pour la danse, ethnologue et sociologue impliqués dans le milieu artistique comme trapéziste et danseur, soulignent la réalité de la douleur (physique mais aussi psychologique, comme l'humiliation par exemple). L'improvisation y est une compétence professionnelle construite, 
permettant de se faire recruter, par un chorégraphe-employeur, et ainsi trouver place sur le marché du travail. Ces perspectives de la sociologie de l'art semblent bien méconnues des enseignant EPS interviewés...

La conception dominante de l'art chez ceux-ci est celle qui caractérise une gestuelle spécifique à l'individu associée à un ressenti propre à chacun, conçue pour être vue par autrui et lui transmettre un message. Appropriation personnelle et créativité y sont deux composantes incontournables du label « artistique ».

Les enseignants évoquent des dilemmes entre apporter/fournir et faire découvrir, ce qui conduit à un guidage apparemment décousu car il dépend des réactions « du jour ». Dans le cadre de ces dilemmes, beaucoup d'enseignants situent la démonstration comme la négation de l'individualisation de l'élève-artiste. La démonstration et la créativité leur semblent incompatibles. La première reste associée à l'idée d'un modèle à copier et à reproduire passivement, la seconde, à l'exploration, la divergence, la spécificité du sujet-artiste. Néanmoins, dans le guidage des élèves vers la construction d'une chorégraphie, la démonstration paraît indispensable à l'enseignant pour asseoir sa crédibilité devant la classe et permettre aux élèves de dépasser leurs inquiétudes initiales face à une activité qu'ils méconnaissent. Ces pratiques effectives dans l'usage de la démonstration, en dépit des réticences affirmées à son égard, illustrent ici les décalages existants entre différents conceptions et approches de l'enseignement artistique, tels que ceux mis en évidence par Lam et Kember (2006).

\title{
Geneviève COGERINO \\ CRIS L-Vis \\ Université Lyon1 \\ genevieve.cogerino@univ-lyon.fr
}

\begin{abstract}
This research aims to understand how the physical education teachers manage their students to perform a dance choreography. The educational procedures studied for this artistic practice are the uses and the designs of the demonstration, and the teacher'contributions to compose and to create. The study is based on fourteen semi-structured interviews with teachers whose levels of expertise varied. The results show that the teachers'and pupils'demonstrations meet many functions, but arouse reluctances despite their proven use. For creation, the processes are guided by pedagogical dilemmas between autonomy and constraints. What implicitly means the art varies according to the teachers'level of expertise.
\end{abstract}

Keywords : dance, PE, creation, demonstration.

\section{Bibliographie}

Arguel M. (1992). « Détournement réciproque dans le domaine gestuel » - in M. Arguel (éd.) Danse : le corps enjeu (34-47). Paris : Presses universitaires de France.

Benhaïm-Grosse P. (2007) «Image du sport scolaire et pratiques d'enseignement au collège et au lycée $»-$ Les Dossiers 190, Ministère de l'Education nationale (DEPP).

Connell J. (2009) « Dance education : an examination of practitioners'perceptions in secondary schools and the necessity for teacher skilled in the pedagogy and content of dance »-Research in Dance Education 10 (115-130). 
Garcia M.-C. (2007) « Représentations « genrées » et sexuation des pratiques circassiennes en milieu scolaire »-Sociétés et Représentations 24 (129-143).

Garcia M.-C. (2013) «Le goût du cirque chez les enseignant d'EPS »-Staps The International Journal of Sport Science and Physical Education 102 (47-60).

Garcia M.-C. \& Vigneron C. (2006). « Le cirque à l'école des banlieues » - Agora Débats Jeunesse 41 (32-48).

Harbonnier-Topin N. \& Barbier J.M. (2014) «L'apprentissage par imitation en danse : une « résonance » constructive ?»-Staps The International Journal of Sport Science and Physical Education 103 (53-68).

Lafont L. \& Martin L. (2014) « Apprentissage et enseignement dans les activités physiques à dimension artistique : considérations théoriques et résultas de recherche »-Staps The International Journal of Sport Science and Physical Education 103 (39-52).

Lam B.-H. \& Kember D. (2006) « The relationship between conceptions of teaching and approaches to teaching $"-$ Teachers and Teaching : Theory and Practice 12 (693-713).

Lemonnier J.-M. (2014). «L'indétermination de la danse à l'école : l'offre socioculturelle face aux enjeux éducatifs » - in : M. Attali et J. SaintMartin (éds.) A l'école du sport : épistémologie du XIX $X^{e}$ siècle à nos jours (137-167). Louvain la Neuve : De Boeck.

Sizorn M. (2008) «Une ethnologue en "Trapézie" : sport, art ou spectacle ?»Ethnologie Française 38 (79-88).

Sorignet P.-E. (2008) «Qu'est-ce que créer?»-Regards sociologiques 35 (37$53)$.

Annexe 1 : Principales caractéristiques des sujets interviewés

Le corpus a été enregistré, transcrit, subdivisé par C. Devillers, A. Gossoin, T. Loyer-Rohault.

\begin{tabular}{lccccc}
\hline Pseudonyme & Sexe & Établissement & Enseigne Danse & Enseigne Cirque & Spécialiste \\
\hline Rémi & H & Clg & non & non & non \\
\hline René & H & LP & non & non & non \\
\hline Rita & F & Lyc & non & non & non \\
\hline Charles & H & Clg & non & oui & moy \\
\hline Carine & F & Clg & non & oui & moy \\
\hline Pierre & H & Clg & oui & non & peu \\
\hline Marie & F & Lyc & oui & non & moy \\
\hline Martin & H & Clg & oui & non & moy \\
\hline Marie-Eve & F & Lyc & oui & non & moy + \\
\hline Éric & H & LP & oui & non & oui \\
\hline Emma & F & LP & oui & non & oui \\
\hline Maryse & F & Clg & oui & oui & moy \\
\hline Marc & H & Clg & oui & oui & moy \\
\hline Édith & F & Clg & oui & oui & oui \\
\hline
\end{tabular}

